\title{
DJ-1 inhibits microglial activation and protects dopaminergic neurons in vitro and in vivo through interacting with microglial
} p65

\author{
Zixuan Lin ${ }^{1}$, Chen Chen ${ }^{1}$, Dongqin Yang ${ }^{1}$, Jianqing Ding ${ }^{2}$, Guanghui Wang $\mathbb{D}^{1 凶}$ and Haigang Ren $\mathbb{D}^{1 凶}$
}

(c) The Author(s) 2021

Parkinson's disease (PD), one of the most common neurodegenerative disorders, is characterized by progressive neurodegeneration of dopaminergic (DA) neurons in the substantia nigra pars compacta (SNpc). DJ-1 acts essential roles in neuronal protection and anti-neuroinflammatory response, and its loss of function is tightly associated with a familial recessive form of PD. However, the molecular mechanism of DJ-1 involved in neuroinflammation is largely unclear. Here, we found that wild-type DJ-1, rather than the pathogenic L166P mutant DJ-1, directly binds to the subunit p65 of nuclear factor-KB (NF-KB) in the cytoplasm, and loss of DJ-1 promotes p65 nuclear translocation by facilitating the dissociation between p65 and NF-KB inhibitor a (IKBa). DJ-1 knockout $\left(D J-1^{-1-}\right)$ mice exhibit more microglial activation compared with wild-type littermate controls, especially in response to lipopolysaccharide (LPS) treatment. In cellular models, knockdown of DJ-1 significantly upregulates the gene expression and increases the release of LPS-treated inflammatory cytokines in primary microglia and BV2 cells. Furthermore, DJ-1 deficiency in microglia significantly enhances the neuronal toxicity in response to LPS stimulus. In addition, pharmacological blockage of NF-KB nuclear translocation by SN-50 prevents microglial activation and alleviates the damage of DA neurons induced by microglial $D J-1$ deficiency in vivo and in vitro. Thus, our data illustrate a novel mechanism by which DJ-1 facilitates the interaction between IKBa and $\mathrm{p} 65$ by binding to $\mathrm{p} 65$ in microglia, and thus repressing microglial activation and exhibiting the protection of DA neurons from neuroinflammation-mediated injury in PD.

Cell Death and Disease (2021)12:715; https://doi.org/10.1038/s41419-021-04002-1

\section{INTRODUCTION}

Parkinson's disease (PD) is one of the most common neurodegenerative diseases, with a prevalence of more than $1 \%$ in the population over 65 years old, and up to $\sim 5 \%$ over age $85[1,2]$. The typical pathological feature of PD is the progressive loss of dopaminergic (DA) neurons, that is selectively detected in the substantia nigra pars compacta (SNpc) [3]. Genetic factors, aging, and neurotoxins contribute to PD pathogenesis [4]. Thus far, more than 20 genes including DJ-1/PARK7 have been identified to be related to multiple forms of familial PD [5]. A great deal of evidence indicates that neuroinflammation-mediated DA neurotoxicity acts a vital role in the pathogenesis of both familial and sporadic forms of PD $[6,7]$.

Microglia are macrophages that reside in the central nervous system (CNS), playing key roles in brain immunity and mediate neuroinflammation in response to neuronal injury or dysfunction [8]. Overactivation of microglia leads to excess production of proinflammatory factors including inducible nitric oxide synthase (iNOS), cyclooxygenase-2 (COX-2), tumor necrosis factor-a (TNFa), prostaglandin E2 (PGE2), interleukin-6 (IL-6), and nitric oxide (NO) [9], which lead to DA neuronal death in PD [10]. A PD-associated protein, DJ-1 homozygous deletion or point mutations including L166P are associated with early-onset autosomal recessive forms of PD [11, 12]. Moreover, altered levels of DJ-1 are also found in sporadic PD patients [13-16]. DJ-1 protein is abundantly expressed in both neurons and glial cells in the CNS, and is mainly distributed in the cytosol and partially in the nucleus and mitochondria [11, 17-19]. It has been demonstrated that DJ-1 protects DA neurons through its multifunctional roles in antioxidative ability, transcriptional regulation, mitochondrial function regulation, and signal transduction in neurons [20]. Recent reports also point out that DJ-1 acts a vital role in the neuroinflammatory response, and the downregulation of DJ-1 augments neuroinflammation in glial cells [21-24]. However, the role of microglial DJ-1 in vivo, as well as the potential molecular mechanisms of DJ-1 in microglia are largely unclear.

Here, we reveal a novel mechanism by which DJ-1 directly binds to p65 in microglial cytoplasm to block neuroinflammation. DJ-1 deficiency facilitates the dissociation between p65 and $\mathrm{I} \mathrm{KBa}$, leading to $\mathrm{p} 65$ nuclear translocation and increases nuclear factor-KB (NF-KB) transcriptional activity. $D J-1^{-1-}$ mice exhibit more microglial activation than wild-type in response to

\footnotetext{
'Jiangsu Key Laboratory of Translational Research and Therapy for Neuropsychiatric disorders \& Department of Pharmacology, College of Pharmaceutical Sciences, Soochow University, 199 Ren'ai Road, Suzhou, Jiangsu 215123, China. ${ }^{2}$ Department of Neurology \& Institute of Neurology, Ruijin Hospital affiliated to Shanghai Jiao Tong University School of Medicine, Shanghai 200025, China. ${ }^{\infty}$ email: wanggh@suda.edu.cn; rhg@suda.edu.cn
} Edited by P. G. Mastroberardino 
A

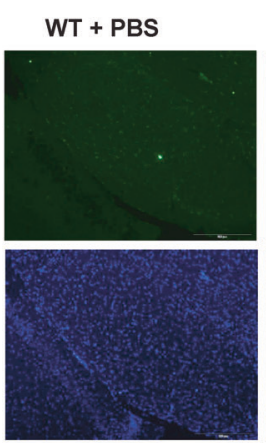

B
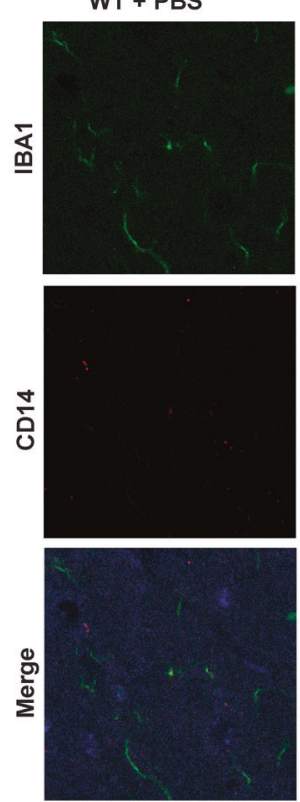

C
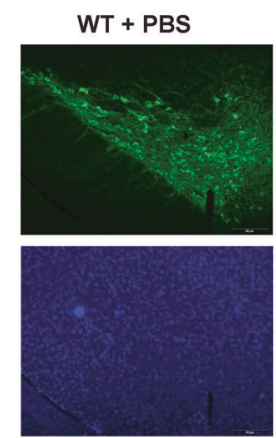

DJ-1 KO + PBS

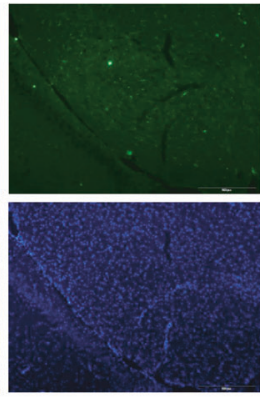

DJ-1 KO + PBS
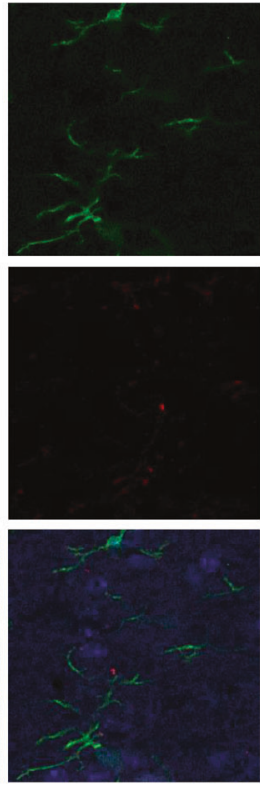

DJ-1 KO + PBS
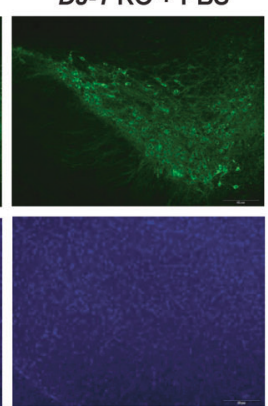

WT + LPS

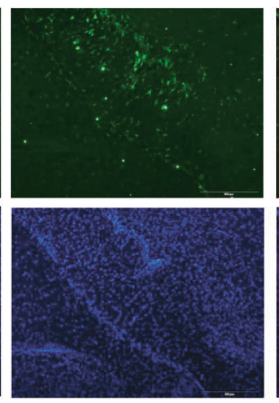

WT + LPS
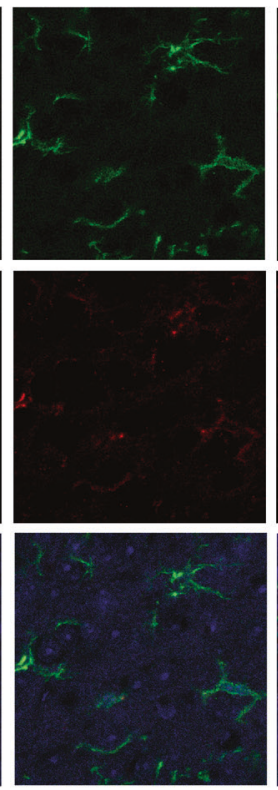

WT + LPS
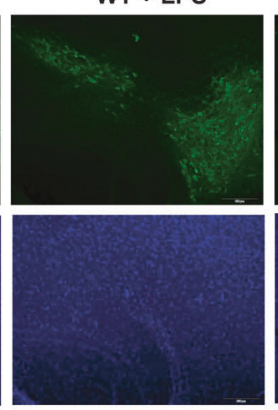

DJ-1 KO + LPS

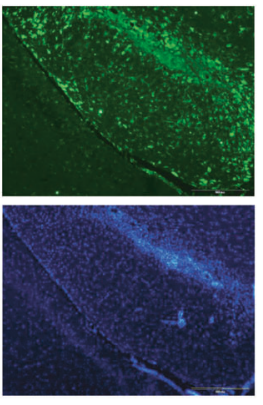

DJ-1 KO + LPS
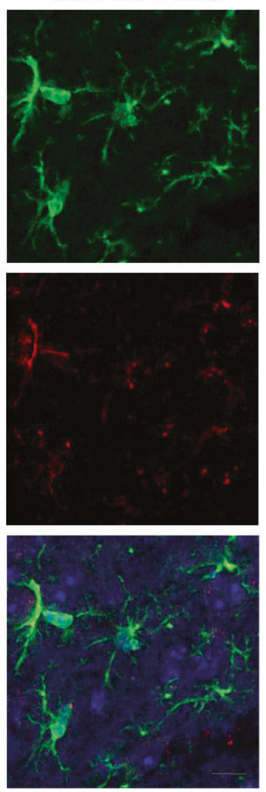

$D J-1 K O+L P S$
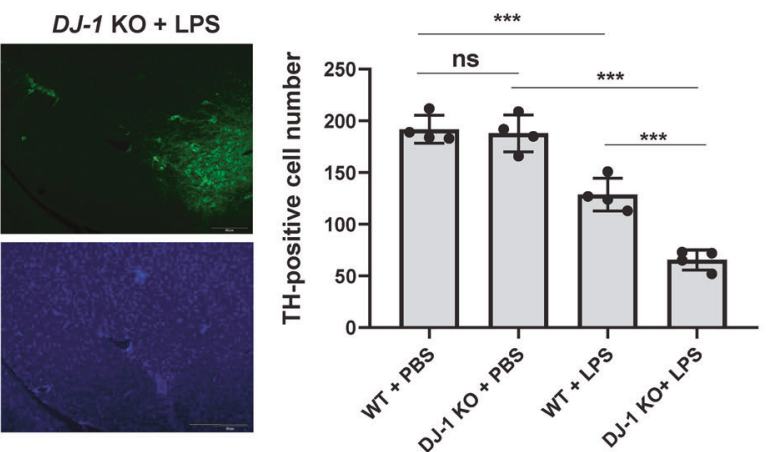

Fig. 1 DJ-1 deficiency leads to microglia activation and DA neuron loss in vivo. A-C DJ-1 ${ }^{-1-}$ and the littermate wild-type controls were microinfused with PBS or LPS into the SN, and 14 days after injection, immunohistochemical staining was conducted. A Slices were stained with anti-IBA1 antibodies. Scale bar, $200 \mu \mathrm{m}$. The fluorescence intensity of IBA1 staining was quantified. $n=4$. B Slices were co-stained with anti-CD14 and anti-IBA1 antibodies. Scale bar, $20 \mu \mathrm{m}$. The fluorescence intensity of CD14 staining was quantified. $n=4$. C Slices were stained with anti-TH antibodies. Scale bar, $200 \mu \mathrm{m}$. TH-positive neuron numbers in the SN of each slice were quantified. $n=4$.

LPS treatment. Moreover, DJ-1 deficiency significantly increases the production of inflammatory factors in microglia and results in DA neuronal loss in response to LPS stimulus. In addition, NF$K B$ inhibitors block microglial activation as well as neuronal cell death induced by DJ-1 deficiency in vivo and in vitro. Thus, our data illustrate a novel mechanism consisting of DJ-1 inhibiting neuroinflammation by facilitating the interaction between IKBa and p65 in microglia.

\section{RESULTS}

\section{DJ-1 deficiency leads to microglial activation and DA neuron} loss

To examine the roles of DJ-1 on microglia in vivo, we first examined the microglia activation in the substantia nigra (SN) in $D J-1^{-1-}$ mice and the littermate wild-type controls, with or without LPS treatment. As shown in Fig. $1 \mathrm{~A}, D J-1^{-1-}$ mice exhibited a greater amount of IBA1 staining than the littermate 
A

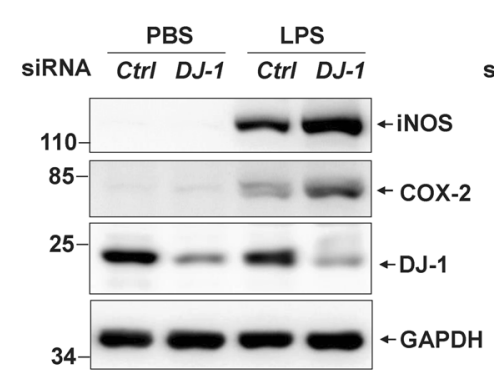

D

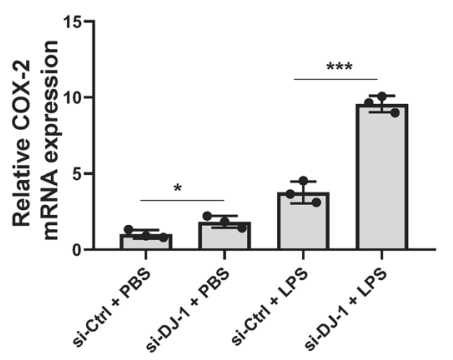

G

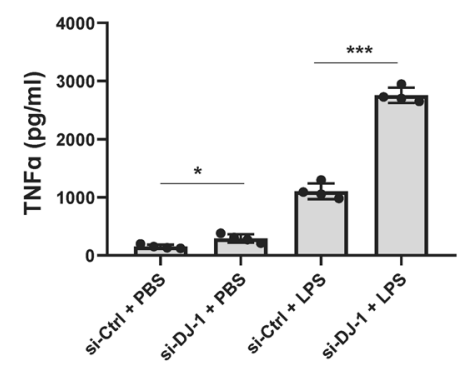

B

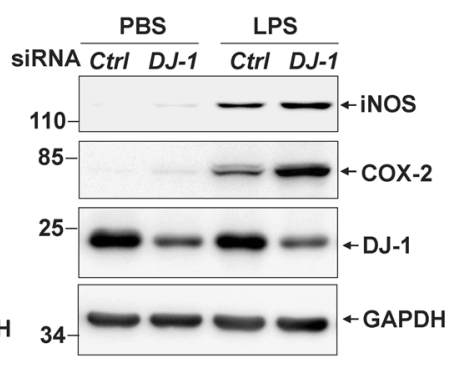

E

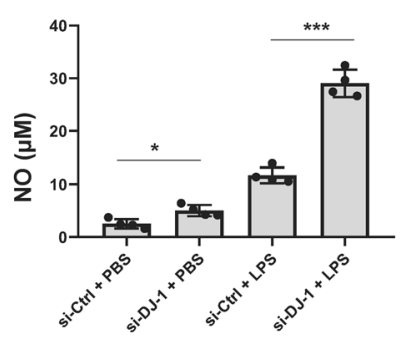

C

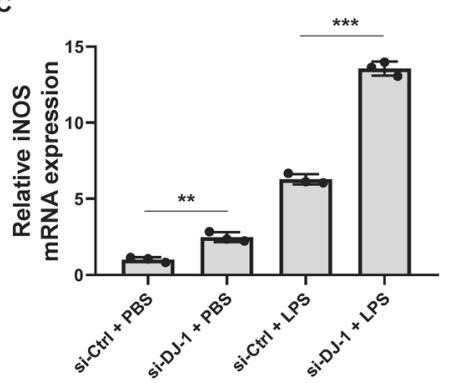

F

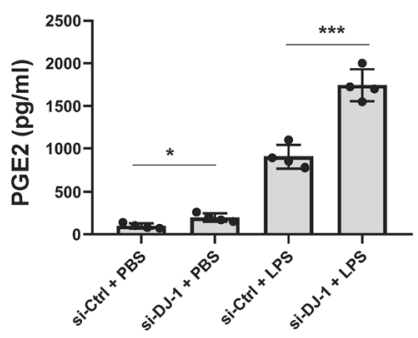

H

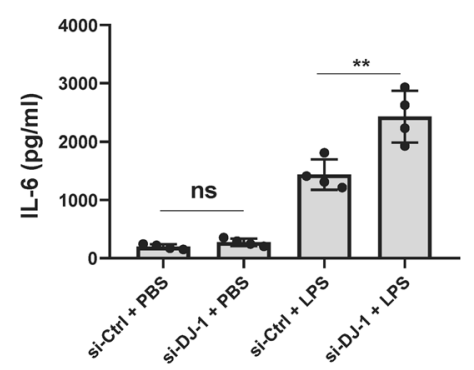

Fig. 2 DJ-1 deficiency results in microglia activation in vitro. A, B si-Ctrl or si-DJ-1 was transfected into primary microglia (A) or BV2 cells (B) for $48 \mathrm{~h}$. The cells were then treated with PBS or LPS $(100 \mathrm{ng} / \mathrm{ml})$ for $24 \mathrm{~h}$. The cell lysates were analyzed by immunoblotting using the indicated antibodies. C, D si-Ctrl or si-DJ-1 was transfected into BV2 cells for $72 \mathrm{~h}$. The cells were then treated with PBS or LPS (100 ng/ml) for $6 \mathrm{~h}$ and then were subjected to qRT-PCR to measure C iNOS or D COX-2 mRNA levels. $n=3$. E-H si-Ctrl or si-DJ-1 was transfected into BV2 cells for $48 \mathrm{~h}$. The cells were then treated with PBS or LPS $(100 \mathrm{ng} / \mathrm{ml})$ for $24 \mathrm{~h}$. The concentration of E NO, F PGE2, G TNF $\alpha$, or H IL-6 in the cultured medium was measured. $n=4$.

wild-type controls, especially in response to LPS treatment. We then examined the expression of CD14, a marker of the proinflammatory phenotype of microglia, to access whether the increased microglia exhibited pro-inflammatory properties. A greater amount of CD14 expression was detected in $D J-1^{-/-}$ mice than that in the littermate wild-type controls. In addition, most of the IBA1-positive microglia were co-labeled with CD14 staining in $D J-1^{-1-}$ mice under LPS treatment (Fig. 1B). Although the number of DA neurons labelled with tyrosine hydroxylase (TH) in the SN of $D J-1^{-1-}$ mice was not significantly different from that of wild-type controls, LPS stimulation significantly reduced the number of DA neurons in $D^{J-1^{-1-}}$ mice compared to wild-type controls (Fig. 1C).

To explore the mechanism by which DJ-1 deficiency causes microglia activation, we performed $D J-1$ siRNA experiments in mouse primary microglia and BV2 microglial cells. We first examined the expression of COX-2 and iNOS, two major inflammatory mediators. Although knockdown of DJ-1 alone induced a slight increase in COX-2 and iNOS expression levels both in primary microglia and BV2 cells (Fig. 2A, B), DJ-1 silencing dramatically increased COX-2 and iNOS levels compared with those in the control in response to LPS stimulation (Fig. 2A, B). In addition, DJ-1 knockdown significantly increased mRNA levels of COX-2 and iNOS, especially in response to LPS treatment (Fig. 2C, D).

Next, we explored the effects of silencing $D J-1$ on the release of inflammatory cytokines using BV2 cells. Inflammatory cytokines NO and PGE2 are key downstream products of iNOS and COX-2, respectively $[25,26]$. DJ-1 knockdown alone slightly increased NO and PGE2 release into the cultured media, whereas DJ-1 deficiency dramatically increased the release of NO and PGE2 in response to LPS stimulation (Fig. 2E, F). In addition, the knockdown of DJ-1 also increased the production of TNFa and IL- 6 under LPS treatment (Fig. 2G, H). These data suggest that the loss of DJ-1 facilitates microglial activation and intrinsically increases the production of various pro-inflammatory cytokines, especially in response to LPS stimulation.

\section{DJ-1 but not its pathogenic L166P mutant represses NF-KB transcriptional activity}

Mitogen-activated protein kinases (MAPKs) including extracellular signal-regulated kinase (ERK), Jun N-terminal kinase (JNK), and p38 are important in the inflammatory response [27], and DJ-1 has been reported to influence their activity in some types of cells such as cancer cells, neurons, or astrocytes [28-32]. Thus, we first tested whether DJ-1 influenced JNK, p38, or ERK1/2 activity in 
A
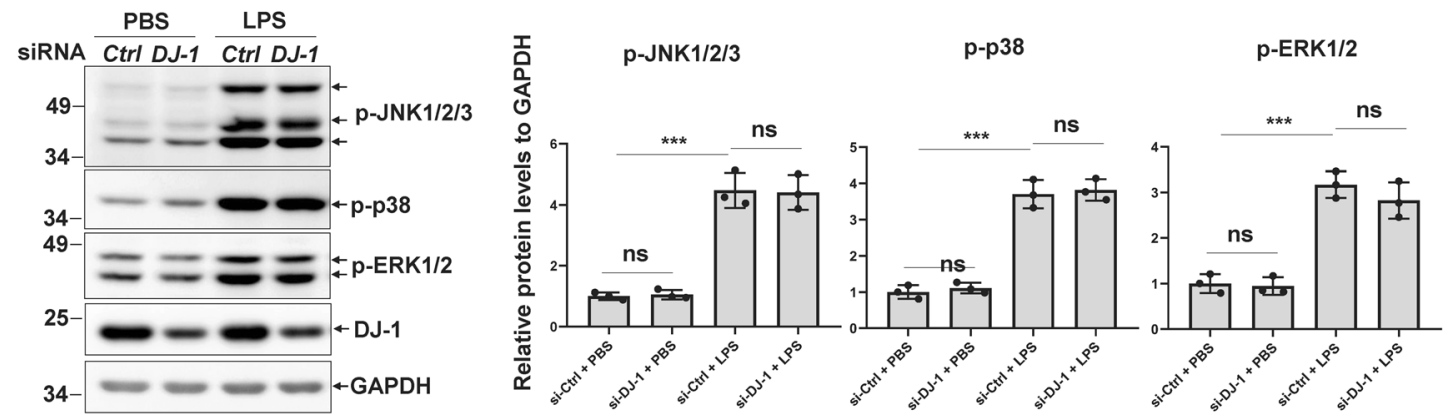

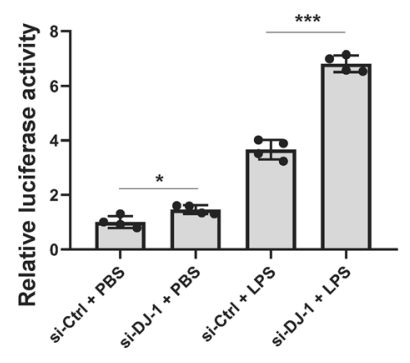

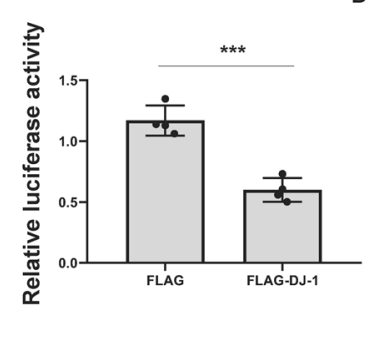

D

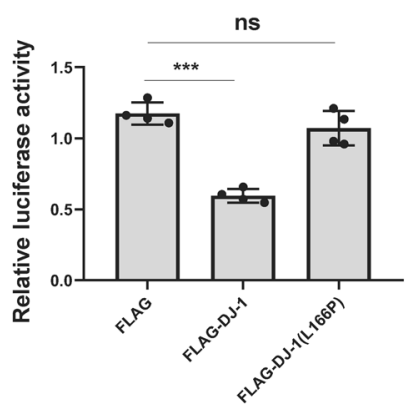

Fig. 3 DJ-1 rather than L166P mutant represses NF-kB transcriptional activity. A si-Ctrl or si-DJ-1 was transfected into BV2 cells for $72 \mathrm{~h}$. Then the cells were treated with PBS or LPS $(100 \mathrm{ng} / \mathrm{ml})$ for $15 \mathrm{~min}$. The cell lysates were analyzed by immunoblotting using the indicated antibodies. The bar graph shows the relative band intensity of $p-J N K 1 / 2 / 3, p-p 38$, or $p$-ERK1/2 to that of GAPDH. $n=3$. B BV2 cells harboring lentiviral NF-kB-luc were transfected with si-Ctrl or si-DJ-1. After $48 \mathrm{~h}$, the cells were treated with PBS or LPS (100 $\mathrm{ng} / \mathrm{ml})$ for $24 \mathrm{~h}$ and then subjected to a luciferase reporter gene assay. $n=4$. C HEK293 cells were transiently transfected with pNF-kB-luc and Renilla along with FLAG or FLAG-DJ-1. After $48 \mathrm{~h}$, the cells were subjected to a luciferase reporter gene assay. One-way ANOVA, $n=4$. D HEK293 cells were transiently transfected with pNF-kB-luc and Renilla along with FLAG, FLAG-DJ-1, or FLAG-DJ-1(L166P). After $48 \mathrm{~h}$, the cells were subjected to a luciferase reporter gene assay. One-way ANOVA, $n=4$.

microglial cells. Although LPS treatment immediately activated JNK, p38, and ERK1/2 phosphorylation, DJ-1 silencing had no significant effect on JNK, p38, or ERK activation, no matter whether BV2 cells were treated with LPS or not (Fig. $3 \mathrm{~A}$ ), suggesting that the effect of DJ-1 on microglial activation is not mediated by the MAPK pathway in microglia.

The expression levels of inflammatory factors are mainly regulated by the transcription factor NF-KB $[25,26]$, and thus, we next examined whether DJ-1 regulates NF-KB activity. In BV2 cells that stably express Cignal lentiviral NF-KB-luciferase, knockdown of DJ-1 alone significantly increased the NF-KB transcription activity (Fig. 3B), and this activation effect was further augmented under LPS stimulation (Fig. 3B).

We next tested the effect of DJ-1 overexpression on NF-kB transcriptional activity. Because the transfection efficiency of overexpression in BV2 cells is very low, we alternatively used HEK293 cells to perform the reporter gene assay. FLAG-DJ-1 overexpression but not the control plasmid significantly repressed NF-KB activity (Fig. 3C). However, the pathogenic DJ-1(L166P) lost the ability to repress NF-KB activity (Fig. 3D). These data indicate that wild-type DJ-1 but not the pathogenic DJ-1(L166P) regulates microglia-mediated neuroinflammation through the NF-KB signaling pathway.

\section{DJ-1 but not the pathogenic L166P mutant binds to the NF-KB subunit p65}

Many studies have shown that DJ-1 can influence transcriptional factors through direct or indirect interactions [33]. We performed co-immunoprecipitation experiments to examine whether DJ-1 interacts with p65, an NF-KB subunit. In HEK293 cells, Flag-DJ-1 could be pulled down by EGFP-p65 rather than EGFP using an anti-GFP antibody (Fig. 4A). Further, endogenous DJ-1 could be co-immunoprecipitated with p65 using anti-p65 antibodies but not control IgG in BV2 cells (Fig. 4B).

We next detected the subcellular distribution of p65 and DJ-1 in cells. DJ-1 and p65 exhibited co-localization in the cytoplasm but not in the nucleus (Fig. 4C). In addition, DJ-1(L166P) exhibited much more mitochondrial localization and less colocalization with p65 in the cytoplasm (Fig. 4C, D). The coimmunoprecipitation experiment also indicated that wild-type DJ-1 but not L166P mutant interacted with p65 in cells (Fig. 4E).

To further identify whether there is a direct interaction between DJ-1 and p65, a GST-pull-down assay using purified recombinant proteins was performed. His-p65 could be directly pulled down by GST-DJ-1 rather than GST or GST-DJ-1(L166P) (Fig. 4F), indicating that wild-type DJ-1 binds to p65 and represses the transcription activity of NF-KB, while the pathogenic L166P mutant DJ-1 loses this ability.

\section{DJ-1 represses p65 nuclear translocation in response to LPS treatment}

We found that DJ-1 interacts with p65 in the cytoplasm and inhibits NF-KB transcription activity (Figs. 3B-D and 4A-E), and p65 nuclear translocation is necessary for NF-KB transcription activity [26]. Therefore, we next examined whether DJ-1 had an effect on p65 nuclear translocation. Knockdown of DJ-1 induced a slight decrease in cytosolic p65 and a slight increase in nuclear p65 (Fig. $5 \mathrm{~A}, \mathrm{~B})$, whereas DJ-1 silencing resulted in a dramatic nuclear translocation of p65 in LPS-treated BV2 cells (Fig. 5A, B).

NF-KB inhibitor a (IKBa) is the major partner that interacts with and sequesters p65 in the cytoplasm, and thus inhibits NF-KB transcription activity, and in response to LPS stimulation, it is phosphorylated by IKK (IKB kinase) and rapidly degraded by the ubiquitin-proteasome system $[25,26]$. Therefore, we examined 
A

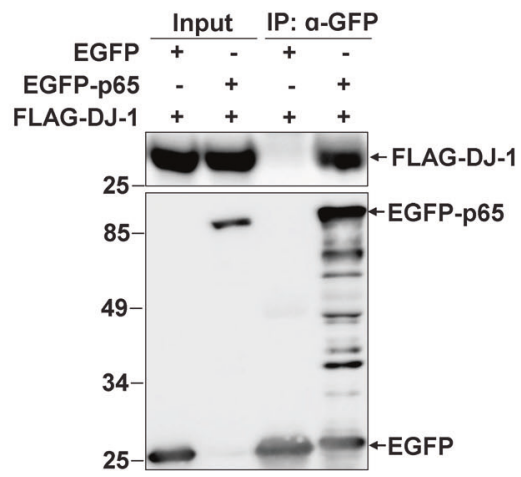

D

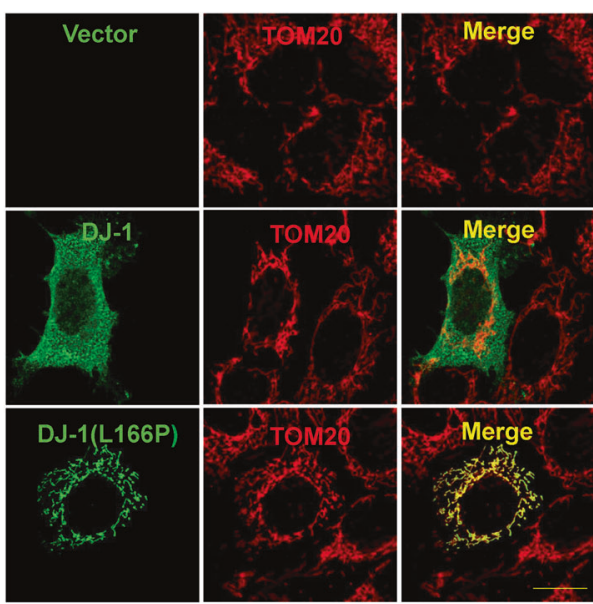

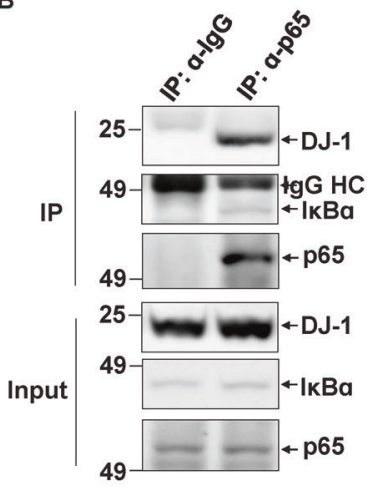

C

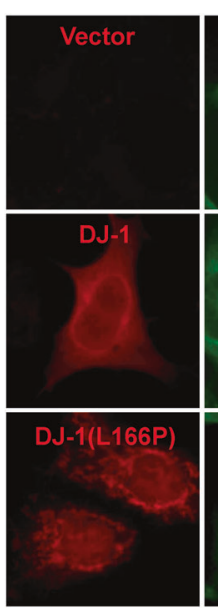

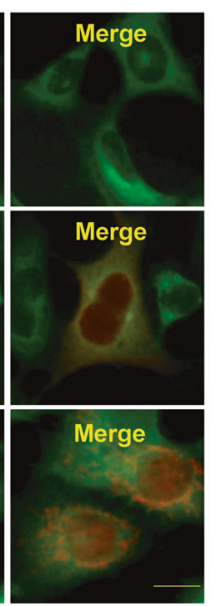

E
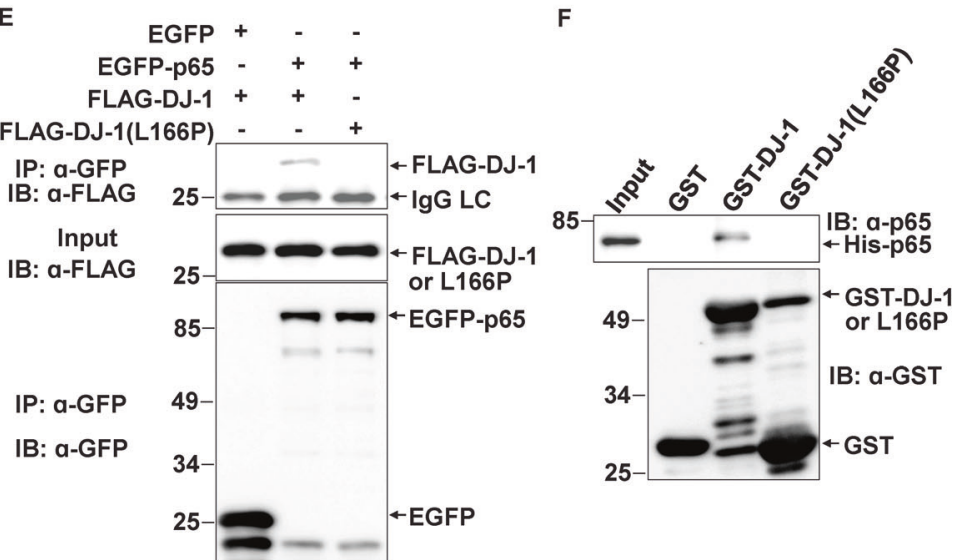

Fig. 4 DJ-1 rather than L166P mutant binds to the p65. A The cell lysate supernatants of HEK293 cells transiently transfected with FLAG-DJ-1 along with EGFP or EGFP-p65 were immunoprecipitated using an anti-GFP antibody. B The supernatants of the BV2 cell lysates were immunoprecipitated using anti-p65 antibodies or normal rabbit lgG. C HEK293 cells transiently transfected with FLAG, FLAG-DJ-1, or FLAG-DJ1(L166P) were subjected to immunocytochemistry using anti-p65 (green) and anti-FLAG (red) antibodies. Scale bar, $10 \mu \mathrm{m}$. D HEK293 cells transiently transfected with FLAG, FLAG-DJ-1, or FLAG-DJ-1(L166P) were subjected to immunocytochemistry using anti-TOM20 (red) and antiFLAG (green) antibodies with a confocal microscope. Scale bar, $10 \mu \mathrm{m}$. E The cell lysate supernatants of HEK293 cells transiently transfected with FLAG-DJ-1 or FLAG-DJ-1(L166P) along with EGFP or EGFP-p65 were subjected to immunoprecipitation with anti-GFP antibodies. F Recombinant GST, GST-DJ-1, or GST-DJ-1(L166P) and His-p65 were subjected to GST-pull-down assay.

whether DJ-1 deletion influences $1 \mathrm{KBa}$ protein levels or the interaction between p 65 and IKBa. However, DJ-1 deletion did not affect IKBa protein levels with or without LPS treatment (Fig. 5C). Interestingly, the knockdown of DJ-1 reduced the interaction between IKBa and p65 in BV2 cells (Fig. 5D). These results indicate that DJ-1 facilitates the interaction between $\mathrm{p} 65$ and $\mathrm{I} \mathrm{KBa}$ by binding to p65, and loss of DJ-1 promotes p65 nuclear translocation and thus activates NF-KB transcription activity.

\section{DJ-1 deficiency increases NF-KB-dependent microglial neurotoxicity}

To further confirm whether microglia activation by DJ-1 deficiency is NF-KB dependent, we examined the inflammatory response in DJ-1-deficient BV2 cells with SN-50, a specific NF-KB inhibitor that directly inhibits NF-kB nuclear transport [34]. SN-50 completely blocked iNOS and COX-2 expression induced by $D J-1$ knockdown under LPS stimulation (Fig. 6A). The secretion of inflammatory cytokines by activated microglia is considered to be toxic to neuronal cells [35]. To determine whether loss of DJ-1 in microglia induces neurotoxicity, we performed propidium iodide (PI) fluorescence staining to determine the toxic effects of loss of microglial DJ-1 on N2a cells. Conditioned media from DJ-1deficient BV2 cells slightly induced N2a cell death (Fig. 6B, C).
Furthermore, conditioned media collected from DJ-1-knockdown BV2 cells in combination with LPS treatment exhibited a greater amount of cell death as compared to that from control siRNAknockdown BV2 cells (Fig. 6B, C). Interestingly, SN-50 significantly inhibited neurotoxicity caused by loss of microglial DJ-1 combined with LPS stimulation (Fig. 6B, C). We also examined whether SN-50 inhibits the augmentation of DJ-1 deficiency-induced TNFa, IL-1 $\beta$, and IL-6 expression in primary microglia in response to LPS stimulation. As shown in Fig. $6 \mathrm{D}, \mathrm{SN}-50$ significantly inhibited the mRNA levels of TNFa, IL-1 $\beta$, and IL- 6 induced by the knockdown of DJ-1 under LPS treatment.

We next wondered whether inhibiting the nuclear translocation of NF-KB blocks microglial activation and alleviates neurotoxicity by loss of microglial DJ-1 in vivo. Stereotactic injection of SN-50 into the SN dramatically inhibited the activation of microglia in $D J$ $1^{-1-}$ mice in response to LPS stimulation (Fig. 6E). SN-50 did not affect the number of DA neurons in both wild-type and the littermate $D J-1^{-1-}$ mice without LPS treatment (Fig. 6F). Whereas, SN-50 treatment dramatically alleviated the loss of DA neurons by LPS in $D J-1^{-1-}$ mice (Fig. 6F). Thus, these data revealed that lack of microglial DJ-1 results in an exaggerated inflammatory response through the promotion of NF-KB nuclear translocation, which increases DA neurotoxicity, and inhibition of NF-KB nuclear 
A

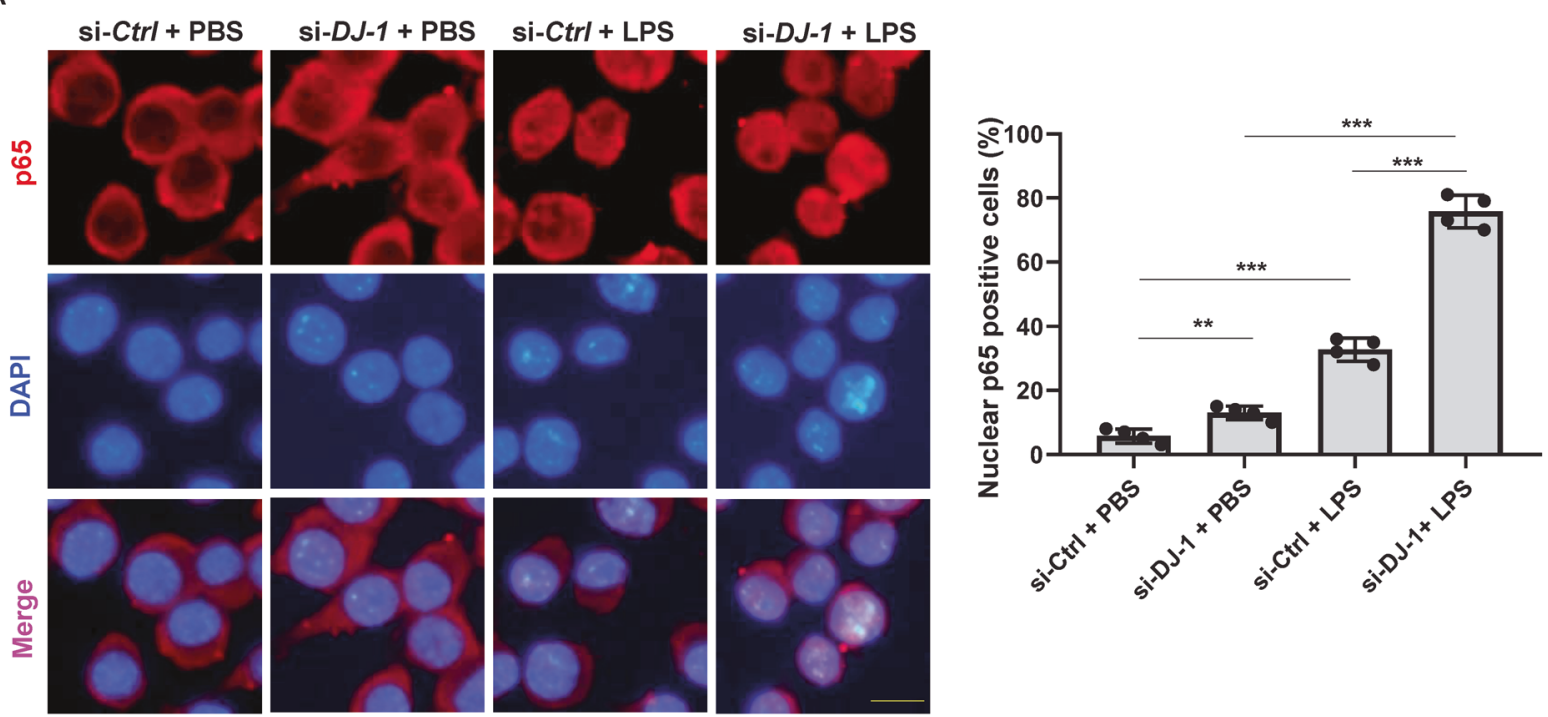

B
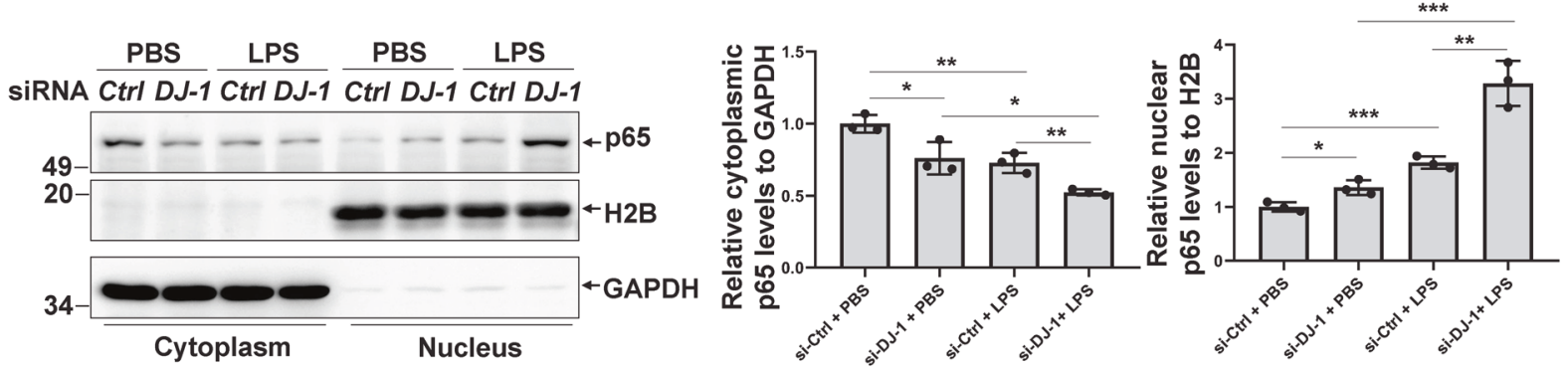

C
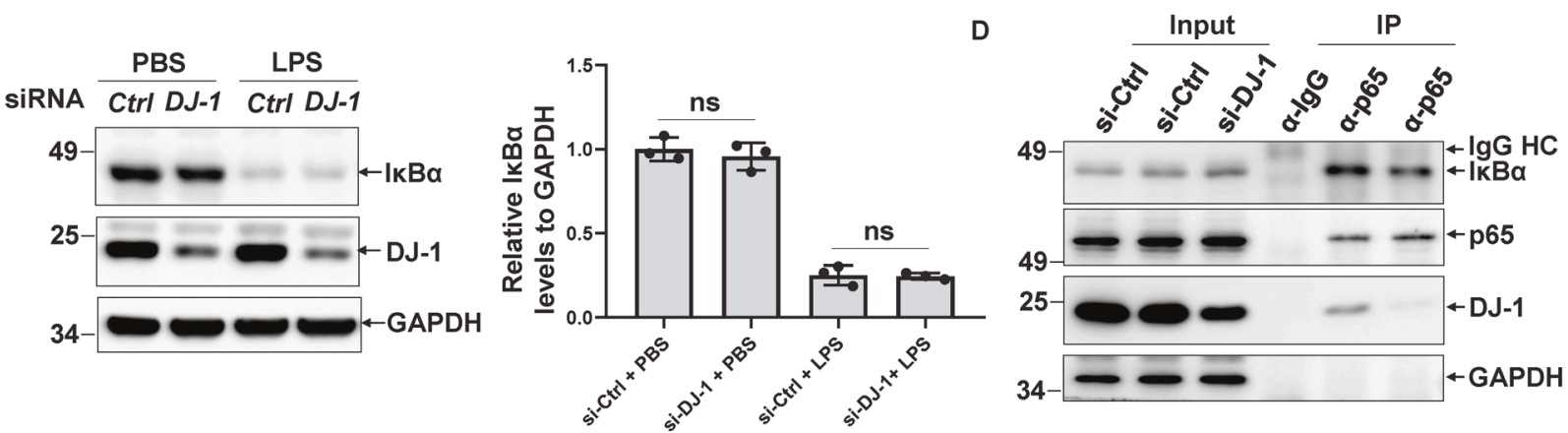

Fig. 5 DJ-1 represses p65 nuclear translocation. A-C BV2 cells were transfected with si-Ctrl or si-DJ-1 for $72 \mathrm{~h}$ and then treated with PBS or LPS $(100 \mathrm{ng} / \mathrm{ml})$ for $15 \mathrm{~min}$. A The cells were stained with anti-p65 antibody and DAPI. Scale bar, $10 \mu \mathrm{m}$. And the percentage of cells with nuclear p 65 distribution was quantified. $n=4$. B The cells were then subjected to a subcellular fractionation assay. The relative band intensity of cytoplasmic or nuclear p65 to that of GAPDH or H2B was quantified, respectively. $n=3$. C Cell lysates were then subjected to immunoblotting. The relative band intensity of $\mathrm{I} \mathrm{KB} \alpha$ to that of GAPDH was quantified. $n=3$. D The supernatants of the BV2 cells transfected with si-Ctrl or si-DJ-1 for $72 \mathrm{~h}$ were immunoprecipitated using anti-p65 antibodies or normal rabbit lgG.

transport is a potential target for alleviating the damage to DA neurons in PD.

\section{DISCUSSION}

Microglia play vital roles in CNS hemostasis and are sensitive to activation in response to signals derived from dysfunctional neurons or neurotoxins. Activated microglia could produce and release pro-inflammatory factors such as TNFa, IL-1a/ $\beta$, and IL-6, leading to damaged DA neurons in the SN [36]. Since it was discovered in 1988 that a large number of activated human leukocyte antigen (HLA)-positive microglia are present in the SNpc of PD patients [37], there has been a dramatically increased focus on the microglia in PD in recent years. Microglia-mediated neuroinflammation is an essential and common pathogenic factor in the early stage of PD and is extensively regarded as a therapeutic target of PD intervention. In addition to the direct damage to DA neurons, microglia-mediated neuroinflammation also induces astrocytes to adopt a neurotoxic function [38].

Recently, it has been reported that the PD-associated genetic factor DJ-1 controls important functions in astrocytes and microglia [21-24]. However, the role of DJ-1 in microglia in vivo and the potential molecular mechanisms of DJ-1 involves in microglial regulation are largely unclear. In the present study, we describe the functions and mechanisms of microglial DJ-1, a PDrelated protein in neuroinflammatory regulation. Wild-type DJ-1 rather than its pathogenic mutant L166P blocks activation of microglia via the NF-KB signaling pathway and protects DA 
A

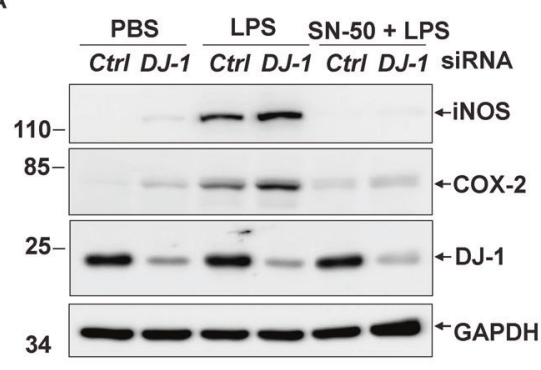

B

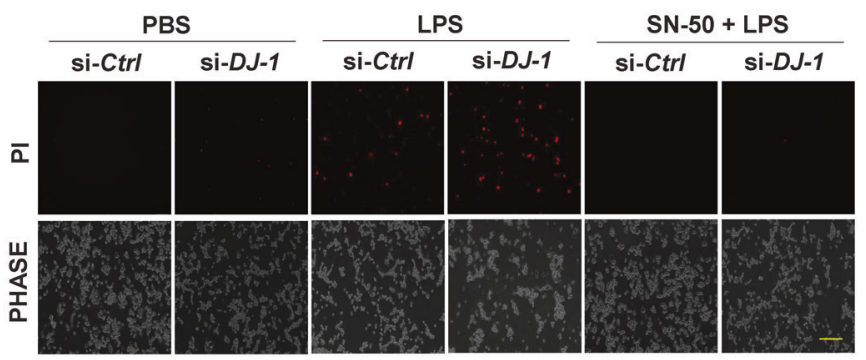

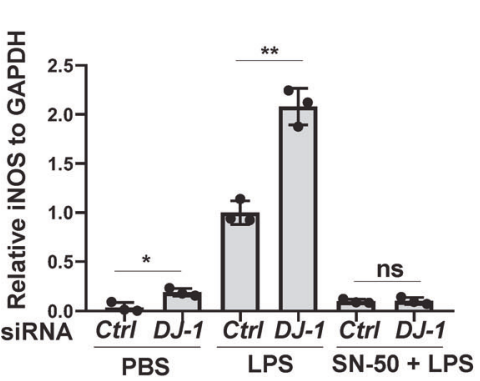

C

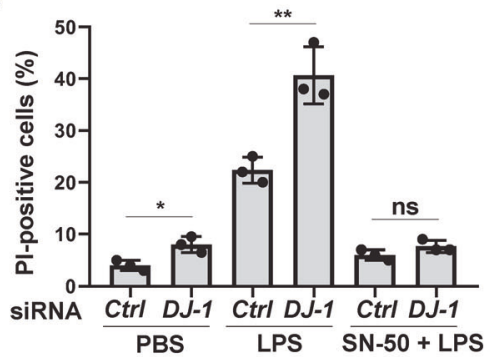

D

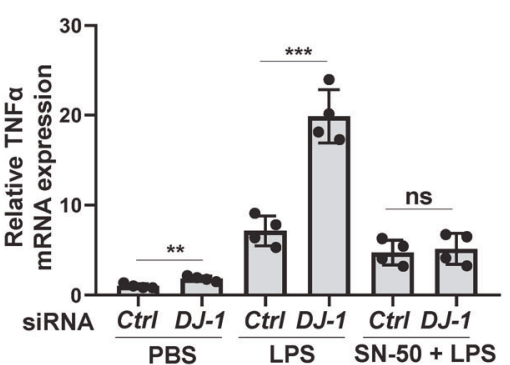

E
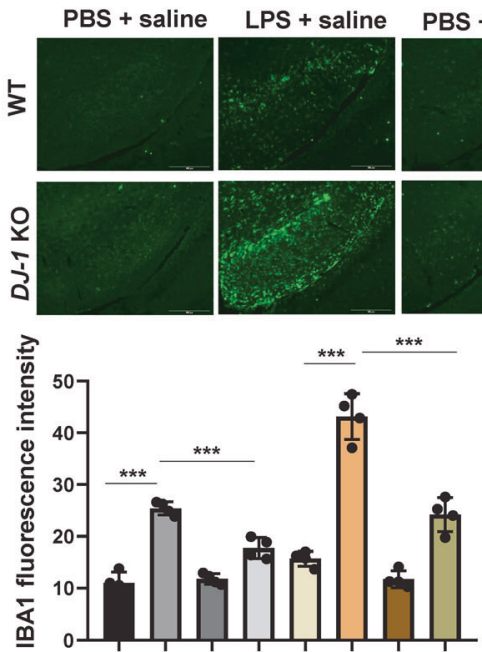

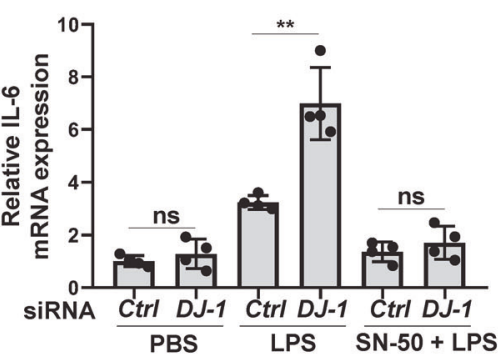

F
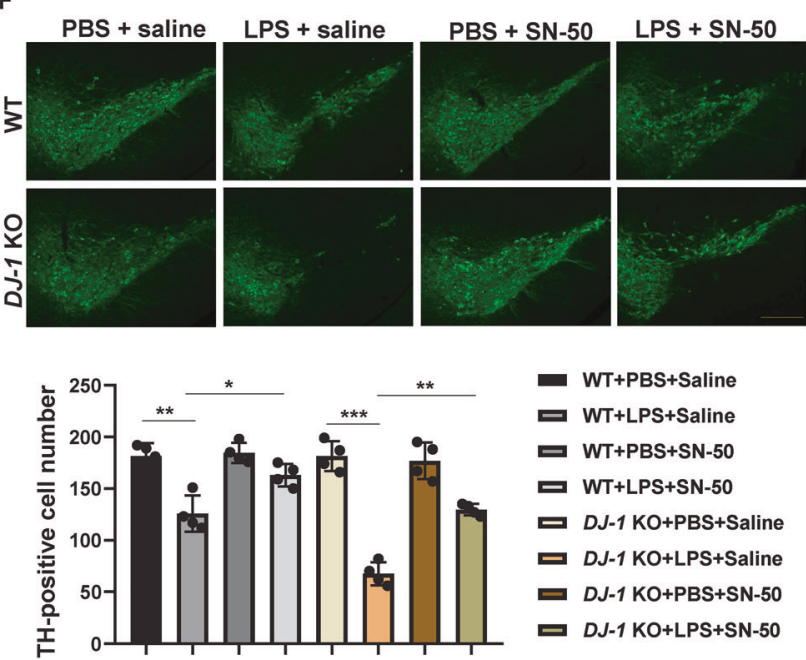

Fig. 6 DJ-1 deficiency increases NF-kB-dependent microglial neurotoxicity. A BV2 cells transfected with si-Ctrl or si-DJ-1 for $48 \mathrm{~h}$ were treated with PBS or LPS $(100 \mathrm{ng} / \mathrm{ml})$ for $24 \mathrm{~h}$ followed by pretreated with SN-50 $(20 \mu \mathrm{M})$ for $1 \mathrm{~h}$ as indicated. After treatment, cell lysates were subjected to immunoblotting. The relative band intensity of iNOS or COX-2 to that of GAPDH was quantified. $n=3$. B N2a cells cultured in conditioned media collected from BV2 cells with the indicated treatment for $24 \mathrm{~h}$ were then stained with PI and visualized with an inverted microscope. Scale bar, $50 \mu \mathrm{m}$. C The percentage of PI-positive cells in (B) was analyzed. $n=3$. D Primary microglia transfected with si-Ctrl or si$D J-1$ for $48 \mathrm{~h}$ were treated with PBS or LPS $(100 \mathrm{ng} / \mathrm{ml})$ for $6 \mathrm{~h}$ followed by pretreated with SN-50 (20 $\mu \mathrm{M})$ for $1 \mathrm{~h}$ as indicated, then the samples were subjected to qRT-PCR. ${ }^{*} P<0.05, n=4$. E, F $D J-1^{-1-}$ and the littermate wild-type controls were microinfused with PBS or LPS for 14 days followed by saline or SN-50 into the SN as indicated, and then the immunohistochemical staining was conducted using (E) anti-IBA1 antibody or (F) anti-TH antibody. The fluorescence intensity of IBA1 (E) and the number of TH-positive neurons (F) in SN of each slice were quantified. $n=4$. 
neurons from inflammatory damage in vitro and in vivo. In our observation, wild-type DJ-1 binds to and sequesters p65 in the cytoplasm, and thus repressing NF-kB hyperactivation, especially under LPS stimulation. Therefore, lacking $D J-1$ in mice, primary microglia and cultured BV2 cells causes a great increase in the proinflammatory phenotype of microglia compared with the controls, especially in response to LPS treatment (Figs. 1B and 2). In addition, it has been reported that DJ-1 has a great antioxidant function and reactive oxygen species (ROS) scavenging capacity $[20,33]$. Interestingly, ROS are crucial for microglial polarization regulation and excessive ROS promote the pro-inflammatory phenotype of microglia [39]. So, we speculated that microglial activation induced by loss of DJ-1 may also be related to intracellular oxidative stress.

Two major transcription factors, NF-KB and activator protein 1 (AP-1) are both involved in microglial activation [40, 41]. MAPKs including ERK, JNK, and p38 are required for AP-1 activation to induce the expression of inflammatory factors [42, 43]. Although DJ-1 has been reported to affect the activity of these kinases in other types of cells [28-32], loss of DJ-1 did not significantly affect their activation in BV2 microglia with or without LPS treatment (Fig. 3A), suggesting that DJ-1 regulation of the inflammatory response is independent of the AP-1 pathway in microglia. In this study, we found that overexpression of DJ-1 inhibits NF-KB activity, and knockdown of DJ-1 activates NF-kB activity (Fig. 3BD). In unstimulated cells, IKB binds to the p65/p50 heterodimer of NF-KB and sequesters NF-KB in the cytosol. Upon stimulation by TNFa, LPS, and ROS, for example, upon LPS recognition, Toll-like receptor 4 (TLR4) undergoes oligomerization, activates its downstream signaling pathway including the IKK complex. Then IKBa is phosphorylated by IKK and subsequently degraded by the ubiquitin-proteasome system, thus leading to a release in NF-KB that is transported into the nucleus and subsequently transactivates its target gene expression such as the expression of iNOS, COX-2, TNFa, IL-1, or IL-6 $[25,26]$.

Here, we found that wild-type DJ-1 rather than its pathogenic mutant L166P directly binds to the p65 subunit and facilitates the interaction between $\mathrm{p} 65$ and $\mathrm{I} \mathrm{KBa}$ in the cytoplasm, as a DJ-1 deficiency leads to a reduction in their interaction (Fig. 5D). Loss of DJ-1 exacerbates the dissociation of p65 and $1 \mathrm{KBa}$, and promotes NF-KB nuclear localization, especially in response to inflammatory stimulation (Fig. 5A, B). Unlike wild-type DJ-1, which is distributed in the cytoplasm, binds to p65 and inhibits NF-KB activity, the L166P mutant DJ-1 translocates to mitochondria and cannot bind to p65 (Fig. 4C-E). It is also possible that the translocation of the L166P mutant DJ-1 to the mitochondria may affect mitochondrial function and promote the activation of microglia by binding to several specific mitochondrial proteins or affecting mitochondrial metabolism, which needs further investigation. Thus, these alterations make microglia harboring L166P mutant DJ-1 more sensitive to inflammatory activators such as LPS, and augments the inflammatory responses, thus aggravating the inflammatory damage to DA neurons. Interestingly, the NF-KB nuclear transport inhibitor $\mathrm{SN}-50$ has a dominant antiinflammatory effect and alleviates the neuroinflammationmediated neurotoxicity induced by DJ-1 deficiency combined with LPS treatment. Many studies have also found that hyperactivation of the NF-KB pathway is closely associated with $P D$ pathogenesis and is considered a promising intervention target for PD [44]. Increased nuclear translocation and transcriptional activity of NF-KB are observed in PD patients as well as in various PD models [41].

In summary, we demonstrated that microglial wild-type DJ-1 but not DJ-1(L166P) represses neuroinflammation and neuroinflammation-mediated neurotoxicity by binding to the p65 subunit of NF-kB, and thus inhibiting NF-kB nuclear translocation and activation (Fig. 7). Loss of DJ-1 functions significantly increases microglia activation and dramatically

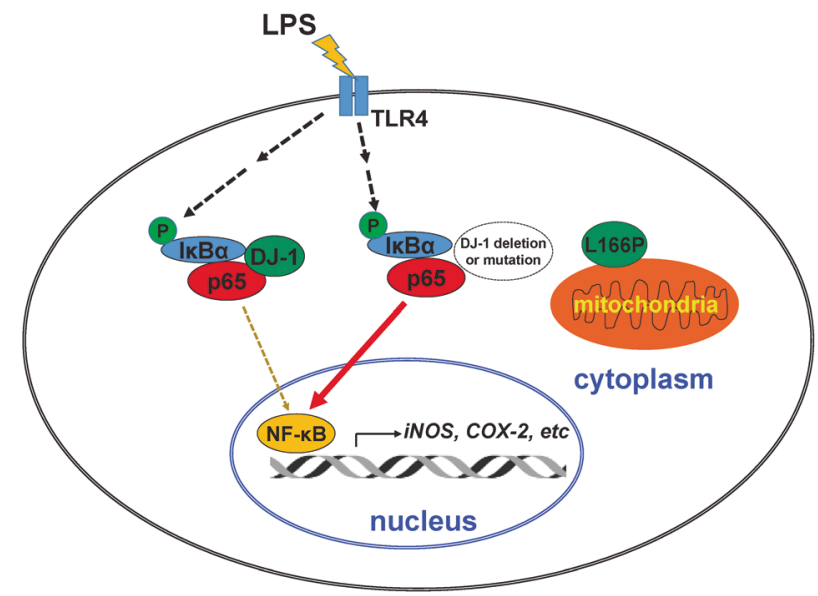

Fig. 7 A schematic diagram shows that DJ-1 functions in neuroinflammation in microglia. Wild-type DJ-1 interacts with the subunit $\mathrm{p} 65$ of $\mathrm{NF}-\kappa \mathrm{B}$ and facilitates the interactions between $1 \kappa \mathrm{B} \alpha$ and $\mathrm{p} 65$, thus weakens LPS-induced NF-KB nuclear transport and microglial activation. Whereas, loss of DJ-1 function by its deletion or L166P mutation which is mainly distributed in mitochondria and fails to bind to p65, augments LPS-induced microglial activation, and aggravates DA neuron damage.

aggregates microglia-mediated neurotoxicity in an NF-kBdependent manner in response to LPS stimulation. In addition, inhibition of NF-KB nuclear translocation inhibits microglial activation and alleviates the loss of DA neurons induced by DJ-1 deficiency in vitro and in vivo.

\section{MATERIALS AND METHODS}

\section{Animals}

$D J-1^{-1-}$ mice were a kind gift from Dr. Jie Shen at Harvard Medical School [45]. The mice were kept in the SPF conditions. All mouse experiments were approved and carried out in accordance with the Regulations of Experimental Animal Administration, which are issued by the Animal Ethics Committee of Soochow University. For drug microfusion in vivo, 6- to 7-week-old mice were anesthetized and then fixed on the stereotaxic apparatus (RWD Life Science, Shenzhen, China). SN-50 (Selleck) was dissolved in $\mathrm{ddH}_{2} \mathrm{O}$ at a concentration of $0.1 \mu \mathrm{g} / \mu \mathrm{l}$ and loaded into a $5-\mu \mathrm{l}$ Hamilton syringe fixed on the stereotaxic apparatus. SN-50 was microinjected into the SN (ML $\pm 1.2 \mathrm{~mm}, \mathrm{AP}-3.3 \mathrm{~mm}$, DV $-4.6 \mathrm{~mm}$ ) at the rate of $0.2 \mu \mathrm{l} / \mathrm{min}(1 \mu \mathrm{L} / \mathrm{mouse})$. LPS (Sigma) $(2 \mathrm{mg} / \mathrm{mL}$ ) was microinjected into the mouse by the same method $(2 \mu \mathrm{l} / \mathrm{mouse})$. Fourteen days after LPS treatment, the mice were perfused with $0.9 \%$ saline, followed by $4 \%$ paraformaldehyde. Then, the brains of mice were removed and post-fixed overnight, followed by incubation with $30 \%$ sucrose solution overnight at $4{ }^{\circ} \mathrm{C}$. Serial $20-\mu \mathrm{M}$-thick slices of midbrain were cut with a frozen microtome for immunohistochemistry. $\mathrm{TH}^{+}$-positive neurons in $\mathrm{SN}$ were counted for each slice under the microscope at a 10x magnification. The fluorescence intensity of IBA1 was analyzed using Photoshop7.0 software (Adobe, CA, USA).

\section{Primary microglia, cell line culture, and transfection}

The procedure for extracting primary microglia from the newborn mouse cortex has been previously described [46]. The mouse microglial BV2 cells [47], mouse neuroblastoma Neuro2a (N2a) cells, and HEK293 cells were cultured in a DMEM medium (Gibco) with $10 \%$ fetal bovine serum, penicillin $(100 \mathrm{U} / \mathrm{ml})$, and streptomycin $(100 \mu \mathrm{g} / \mathrm{ml})$. All of the cell lines are authenticated by STR profiling. The small interfering RNAs (siRNAs) and the expression plasmids were transfected into cells with Lipofectamine RNAiMAX reagent (Invitrogen) and Lipofectamine2000 reagent (Invitrogen), respectively.

\section{Immunohistochemistry and immunocytochemistry}

For immunohistochemistry, the slices of mouse midbrains were stained overnight with anti-IBA1 antibodies (019-19741, Wako, Japan) for labelling microglia, and anti-TH antibodies (AB152, Millipore) for DA neurons, and then incubated with the Alexa Fluor 488-conjugated fluorescent secondary 
antibody for $1 \mathrm{~h}$. And anti-CD14 (60253-1-lg, Proteintech Technology) antibody and the Alexa Fluor 594-conjugated secondary antibody (Invitrogen) were used for pro-inflammatory microglia staining. Then, the slices were labelled with 4',6-diamidino-2-phenylindole (DAPI, Sigma) for $10 \mathrm{~min}$ to visualize cellular nuclei. For immunocytochemistry, fixed HEK293 cells were incubated with polyclonal anti-p65 (ab32536, Abcam) or antiTOM20 (42406, Cell Signaling Technology) with monoclonal anti-FLAG (F3165, Sigma) antibodies for $4 \mathrm{~h}$, and then incubated with Alexa Fluor 594and 488-conjugated fluorescent secondary antibody for $2 \mathrm{~h}$. For fixed BV2 cells, the primary polyclonal anti-p65 and Alexa Fluor 594-conjugated fluorescent secondary antibodies were applied, and then DAPI was used to stain the nuclei. Finally, the slices and cells were visualized using an IX71 inverted system microscope (Olympus, Japan), or an LSM800 confocal microscope (Zeiss, Germany).

\section{Plasmids, siRNAs, and drugs}

The p3xFLAG-myc-cmv-24-DJ-1 and DJ-1(L166P), pGEX-5x-1-DJ-1 and DJ-1 (L166P) plasmids have been previously described $[19,48]$. The EGFP-p65 plasmid was a kind gift from Dr. Yizheng Wang. pET-15b-p65 has been previously described [49]. The pNF-KB-luc-containing quadruple NF-KB response element (GGGAATTCC) was purchased from Beyotime Biotechnology Co. Ltd. (Nantong, China). Cignal lentiviral NF-KB-luc was purchased from Qiagen. The plasmid fidelities were identified by sequencing. siRNA sequences targeting mouse $D J-1$ mRNA were as follows: sense: $5^{\prime}$ -CGCUUGUUCUCAAAGACUATT-3', anti-sense: $5^{\prime}$-UAGUCUUUGAGAACAAG CGGT-3'.

\section{Immunoprecipitation assay and GST-pull-down assay}

The procedures of immunoprecipitation assay using polyclonal anti-GFP antibodies (Roche), polyclonal anti-p65 antibodies (Cell Signaling Technology), and normal rabbit IgG (Santa Cruz Biotechnology) were according to our previous work [19]. Bound proteins and cell lysates (input) were analyzed by immunoblot using the indicated primary antibodies and the light chain-specific secondary antibodies (Jackson). For the pull-down assay, GST, GST-DJ-1, and GST-DJ-1(L166P) $(20 \mu \mathrm{g})$ expressed in Escherichia coli strain JM109 were incubated with glutathione-Sepharose 4B (GE Healthcare) for $30 \mathrm{~min}$ in an ice bath, respectively. After washing three times, the beads were incubated with His-p65 $(50 \mu \mathrm{g})$, which is expressed by Escherichia coli strain BL21 in an ice bath for $4 \mathrm{~h}$. Subsequently, the beads were washed with cold PBS five times. The input representing 10\% of the proteins used in the immunoprecipitation or pull-down assay and the interacting proteins were analyzed by immunoblotting.

\section{Immunoblotting and antibodies}

Cells were dissolved in cell lysis buffer $(0.5 \%$ deoxycholate, $1 \%$ NP-40, $50 \mathrm{mM}$ Tris- $\mathrm{HCl} \mathrm{pH} 7.5,150 \mathrm{mM} \mathrm{NaCl}$, and protease inhibitor cocktail (Roche)). The samples were separated by 10 or $15 \%$ SDS-PAGE and then transferred onto a polyvinylidene difluoride membrane (PVDF). The PVDF membranes were incubated with the primary antibodies as follows overnight at $4{ }^{\circ} \mathrm{C}$ : polyclonal anti-DJ-1 antibody (AB9212, Chemicon), monoclonal anti-GAPDH antibody (MAB374, Millipore), polyclonal antiiNOS (ab15323), polyclonal anti-COX2 (ab15191), polyclonal anti-p65 (ab32536) and polyclonal anti-H2B (ab45695) antibodies (Abcam), polyclonal anti-IKBa antibody (4812, Cell Signaling Technology), monoclonal anti-FLAG antibody (F3165, Sigma), and monoclonal anti-DJ-1 (sc-55573) and anti-GFP (sc-9996) antibody (Santa Cruz). The secondary antibodies, consisting of horseradish peroxidase-conjugated sheep anti-mouse or antirabbit antibodies (Amersham Pharmacia Biotech), were used for visualization with enhanced chemiluminescence $(E C L)$ detection kit (Amersham Biosciences) and the use of a Chemiluminescence Imaging System (Bioshine ChemiQ 4800).

\section{ELISA assay and NO measurement}

The levels of PGE2 (Cayman Chemical Company), TNFa, and IL-6 (Boster Biological Technology) in $100 \mu \mathrm{l}$ of BV2 cultured media were detected using enzyme-linked immunosorbent assay (ELISA) kits. The NO concentration was measured using the Griess method with an NO assay kit (Beyotime Biotechnology) according to the manufacturer's instructions.

\section{Quantitative real-time PCR (qRT-PCR)}

Total RNA was extracted from BV2 cells or primary microglia using TRIzol reagent (Invitrogen). Then, the RNA was reverse-transcribed into cDNA using PrimeScript RT Master Mix (Takara). Subsequently, quantitative measurement of the target mRNA was subjected to qRT-PCR with SYBR Green Real-Time PCR Master Mix within a 7500 real-time PCR system (Applied Biosystems). The following primers were used: mouse COX-2, 5'CAGGCTGAACTTCGAAACA- $3^{\prime}$ and $5^{\prime}$-GCTCACGAGGCCACTGATACCTA- ${ }^{\prime}$; mouse iNOS, $5^{\prime}$-TCCCAGCCTGCCCCTTCAAT- $3^{\prime}$ and $5^{\prime}$-CGGATCTCTCTCC TCCTGGG-3'; mouse IL-6, 5'-TAGTCCTTCCTACCCCAATTTCC-3' and $5^{\prime}-T$ GGTCCTTAGCCACTCCTTC-3'; mouse IL-1 $\beta, 5^{\prime}$-GCAACTGTTCCTGAACTCAA CT- $3^{\prime}$ and $5^{\prime}$-ATCTITTGGGGTCCGTCAACT- $3^{\prime}$; and mouse TNFa, $5^{\prime}$-CCCTCACACTCAGATCATCTTCT-3' and 5'-GCTACGACGTGGGCTACAG-3'; mouse $\beta$-actin, $5^{\prime}$-GACCTGACTGACTACCTC-3' and $5^{\prime}$-GACAGCGAGGCCAGGATG$3^{\prime}$. The relative mRNA levels of these genes to $\beta$-actin were calculated by the $2^{-\Delta \Delta C T}$ method.

\section{Nuclear and cytoplasmic fractionation assay}

The procedure for extracting nuclear and cytoplasmic fractionation has been previously described [50]. Briefly, the BV2 cells were collected and dissolved in fractionation buffer $(2 \mathrm{mM} \mathrm{MgAc}, 3 \mathrm{mM} \mathrm{CaCl}, 320 \mathrm{mM}$ sucrose, $1 \mathrm{mM}$ dithiothreitol (DTT), $0.1 \mathrm{mM}$ EDTA, 0.5\% NP-40, and $0.5 \mathrm{mM}$ phenylmethylsulfonyl fluoride (PMSF)) on ice for $20 \mathrm{~min}$. After centrifugation at $600 \times g$ at $4{ }^{\circ} \mathrm{C}$ for $15 \mathrm{~min}$, the collected supernatant was the cytoplasmic fraction. The collected pellet was washed once using fractionation buffer without NP-40, and then dissolved in nuclear buffer (25\% glycerol, $20 \mathrm{mM}$ HEPES (pH 7.9), $1.5 \mathrm{mM} \mathrm{MgCl}, 0.2 \mathrm{mM}$ EDTA, $280 \mathrm{mM} \mathrm{KCl}, 1 \mathrm{mM}$ DTT, 0.3\% NP-40, and $0.5 \mathrm{mM}$ PMSF) as the nuclear fraction.

\section{Luciferase reporter gene assay}

BV2 cells stably expressing Cignal lentiviral NF-KB-luc were constructed by limited dilution method and selected with puromycin $(2.5 \mu \mathrm{g} / \mathrm{ml})$ (Invitrogen) following transfection. BV2 cells stably expressing Cignal lentiviral NF-KB-luc were transfected with si-Ctrl or si-DJ-1. After $48 \mathrm{~h}$, the cells were treated with $100 \mathrm{ng} / \mathrm{ml}$ of LPS or the equal volume PBS for $24 \mathrm{~h}$. In HEK293 cells, cells were transfected with FLAG-DJ-1, FLAG-DJ-1(L166P), or empty vector, along with pNF-KB-luc and the Renilla luciferase vector $\mathrm{pRL}-\mathrm{CMV}$, which acts as a normalized control. After $48 \mathrm{~h}$, firefly and Renilla luciferase activities were detected with a dual-luciferase reporter system (Promega) using a microplate reader (Infinite M1000 Pro, Tecan).

\section{Conditioned medium assays}

The procedure for conditioned medium assays has been previously described [51]. Briefly, si-Ctrl or si-DJ-1 were transfected into BV2 cells for $72 \mathrm{~h}$, and then cells were pretreated with or without SN-50 $(20 \mu \mathrm{M})$ for $1 \mathrm{~h}$, followed by treatment with or without LPS $(100 \mathrm{ng} / \mathrm{ml}$ ) (Sigma) for $24 \mathrm{~h}$. The cells were then washed twice with PBS and cultured for another $24 \mathrm{~h}$ in fresh media to generate the conditioned media. The conditioned media of BV2 cells was then collected and filtered by a $0.22 \mu \mathrm{m}$ filter to culture $\mathrm{N} 2 \mathrm{a}$ cells for $24 \mathrm{~h}$. The N2a cells were then stained with $1 \mu \mathrm{M}$ PI (Sigma) and visualized with an inverted microscope IX71 (Olympus, Japan). The PIpositive cells were counted and analyzed.

\section{Statistical analysis}

The densitometric values of three independent immunoblotting experiments were calculated by Photoshop 7.0 software (Adobe). The data presented as the mean $\pm S D$ from at least three independent experiments were analyzed by GraphPad Prism 8.0 software (San Diego, CA, USA). The statistical significance of the differences between groups was determined using $t$-test (two-tailed) following two-way ANOVA unless otherwise specified in the figure legends. The criterion of significance was set at $P$ $<0.05$. In this study, $P$ values less than 0.001 were shown as ${ }^{* * *} P<0.001, P$ values less than 0.01 were shown as ${ }^{* *} P<0.01, P$ values less than 0.05 were shown as * $P<0.05$, and no statistical significance was shown as "ns". The sample size of experiments is determined on basis of literature in this field. No sample was excluded from the analysis. Animals were carefully assigned based on genotype and age, rather than randomly. There were no studies in which investigators were blinded. The replicate numbers are described in each figure legend.

\section{REFERENCES}

1. de Lau LM, Breteler MM. Epidemiology of Parkinson's disease. Lancet Neurol. 2006;5:525-35. 
2. Li T, Le W. Biomarkers for Parkinson's disease: how good are they? Neurosci. Bull. 2020;36:183-94.

3. Poewe W, Seppi K, Tanner CM, Halliday GM, Brundin P, Volkmann J, et al. Parkinson disease. Nat Rev Dis Prim. 2017;3:17013.

4. Pang SY, Ho PW, Liu HF, Leung CT, Li L, Chang EES, et al. The interplay of aging, genetics and environmental factors in the pathogenesis of Parkinson's disease. Transl Neurodegener. 2019;8:23.

5. Wang $R$, Sun $H$, Wang $G$, Ren $H$. Imbalance of lysine acetylation contributes to the pathogenesis of Parkinson's disease. Int J Mol Sci. 2020;21:7182.

6. Wang Q, Liu Y, Zhou J. Neuroinflammation in Parkinson's disease and its potential as therapeutic target. Transl Neurodegener. 2015;4:19.

7. Kam TI, Hinkle JT, Dawson TM, Dawson VL. Microglia and astrocyte dysfunction in parkinson's disease. Neurobiol Dis. 2020;144:105028.

8. Aloisi F. Immune function of microglia. Glia. 2001;36:165-79.

9. Block ML, Zecca L, Hong JS. Microglia-mediated neurotoxicity: uncovering the molecular mechanisms. Nat Rev Neurosci. 2007;8:57-69.

10. Hirsch EC, Hunot S. Neuroinflammation in Parkinson's disease: a target for neuroprotection? Lancet Neurol. 2009;8:382-97.

11. Bonifati V, Rizzu P, van Baren MJ, Schaap O, Breedveld GJ, Krieger E, et al. Mutations in the DJ-1 gene associated with autosomal recessive early-onset parkinsonism. Science. 2003;299:256-9.

12. Abou-Sleiman PM, Healy DG, Quinn N, Lees AJ, Wood NW. The role of pathogenic DJ-1 mutations in Parkinson's disease. Ann Neurol. 2003;54:283-6.

13. Waragai M, Wei J, Fujita M, Nakai M, Ho GJ, Masliah E, et al. Increased level of DJ-1 in the cerebrospinal fluids of sporadic Parkinson's disease. Biochem Biophys Res Commun. 2006;345:967-72.

14. Hong Z, Shi M, Chung KA, Quinn JF, Peskind ER, Galasko D, et al. DJ-1 and alphasynuclein in human cerebrospinal fluid as biomarkers of Parkinson's disease. Brain. 2010;133:713-26.

15. Devic I, Hwang H, Edgar JS, Izutsu K, Presland R, Pan C, et al. Salivary alphasynuclein and DJ-1: potential biomarkers for Parkinson's disease. Brain. 2011;134: e178.

16. Shi M, Bradner J, Hancock AM, Chung KA, Quinn JF, Peskind ER, et al. Cerebrospinal fluid biomarkers for Parkinson disease diagnosis and progression. Ann. Neurol. 2011;69:570-80.

17. Canet-Avilés RM, Wilson MA, Miller DW, Ahmad R, McLendon C, Bandyopadhyay $S$, et al. The Parkinson's disease protein DJ-1 is neuroprotective due to cysteinesulfinic acid-driven mitochondrial localization. Proc Natl Acad Sci USA. 2004;101:9103-8.

18. Zhang L, Shimoji M, Thomas B, Moore DJ, Yu SW, Marupudi NI, et al. Mitochondrial localization of the Parkinson's disease related protein DJ-1: implications for pathogenesis. Hum Mol Genet. 2005;14:2063-73.

19. Ren H, Fu K, Wang D, Mu C, Wang G. Oxidized DJ-1 interacts with the mitochondrial protein BCL-XL. J Biol Chem. 2011;286:35308-17.

20. Repici M, Giorgini F. DJ-1 in Parkinson's disease: clinical insights and therapeutic perspectives. J Clin Med. 2019;8:1377.

21. Kim JH, Choi DJ, Jeong HK, Kim J, Kim DW, Choi SY, et al. DJ-1 facilitates the interaction between STAT1 and its phosphatase, SHP-1, in brain microglia and astrocytes: a novel anti-inflammatory function of DJ-1. Neurobiol. Dis. 2013;60:1-10.

22. Trudler D, Weinreb O, Mandel SA, Youdim MB, Frenkel D. DJ-1 deficiency triggers microglia sensitivity to dopamine toward a pro-inflammatory phenotype that is attenuated by rasagiline. J Neurochem. 2014;129:434-47.

23. Ji YJ, Wang HL, Yin BL, Ren XY. Down-regulation of DJ-1 augments neuroinflammation via Nrf2/Trx1/NLRP3 axis in MPTP-induced Parkinson's disease mouse model. Neuroscience. 2020;442:253-63.

24. Peng L, Zhou Y, Jiang N, Wang T, Zhu J, Chen $Y$, et al. DJ-1 exerts antiinflammatory effects and regulates NLRX1-TRAF6 via SHP-1 in stroke. J Neuroinflammation. 2020;17:81.

25. Oeckinghaus A, Ghosh S. The NF-kappaB family of transcription factors and its regulation. Cold Spring Harb Perspect Biol. 2009;1:a000034.

26. Napetschnig J, Wu H. Molecular basis of NF-kappaB signaling. Annu Rev Biophys. 2013;42:443-68.

27. Sabio G, Davis RJ. TNF and MAP kinase signalling pathways. Semin Immunol. 2014;26:237-45.

28. Mo JS, Kim MY, Ann EJ, Hong JA, Park HS. DJ-1 modulates UV-induced oxidative stress signaling through the suppression of MEKK1 and cell death. Cell Death Differ. 2008;15:1030-41.

29. Waak J, Weber SS, Waldenmaier A, Görner K, Alunni-Fabbroni M, Schell H, et al. Regulation of astrocyte inflammatory responses by the Parkinson's diseaseassociated gene DJ-1. FASEB J. 2009;23:2478-89.

30. Mo JS, Jung J, Yoon JH, Hong JA, Kim MY, Ann EJ, et al. DJ-1 modulates the p38 mitogen-activated protein kinase pathway through physical interaction with apoptosis signal-regulating kinase 1. J Cell Biochem. 2010;110:229-37.

31. Takahashi-Niki K, Kato-Ose I, Murata H, Maita H, Iguchi-Ariga SMM, Ariga $H$. Epidermal growth factor-dependent activation of the extracellular signal- regulated kinase pathway by DJ-1 protein through its direct binding to c-Raf protein. J Biol Chem. 2015;290:17838-47.

32. Xu X, Wang R, Hao Z, Wang G, Mu C, Ding J, et al. DJ-1 regulates tyrosine hydroxylase expression through CaMKKbeta/CaMKIV/CREB1 pathway in vitro and in vivo. J Cell Physiol. 2020;235:869-79.

33. Ariga H, Takahashi-Niki K, Kato I, Maita H, Niki T, Iguchi-Ariga SM. Neuroprotective function of DJ-1 in Parkinson's disease. Oxid Med Cell Longev. 2013;2013:683920.

34. Lin YZ, Yao SY, Veach RA, Torgerson TR, Hawiger J. Inhibition of nuclear translocation of transcription factor NF-kappa B by a synthetic peptide containing a cell membrane-permeable motif and nuclear localization sequence. J Biol Chem. 1995:270:14255-8.

35. Glass CK, Saijo K, Winner B, Marchetto MC, Gage FH. Mechanisms underlying inflammation in neurodegeneration. Cell. 2010;140:918-34.

36. Yang L, Mao K, Yu H, Chen J. Neuroinflammatory responses and Parkinson' disease: pathogenic mechanisms and therapeutic targets. J Neuroimmune Pharm. 2020;15:830-7.

37. McGeer PL, Itagaki $\mathrm{S}$, Boyes $\mathrm{BE}, \mathrm{McGeer} \mathrm{EG}$. Reactive microglia are positive for HLA-DR in the substantia nigra of Parkinson's and Alzheimer's disease brains. Neurology. 1988;38:1285-91.

38. Liddelow SA, Guttenplan KA, Clarke LE, Bennett FC, Bohlen CJ, Schirmer L, et al. Neurotoxic reactive astrocytes are induced by activated microglia. Nature. 2017:541:481-7.

39. Tan HY, Wang N, Li S, Hong M, Wang $X$, Feng $Y$. The reactive oxygen species in macrophage polarization: reflecting its dual role in progression and treatment of human diseases. Oxid Med Cell Longev. 2016;2016:2795090.

40. Shabab T, Khanabdali R, Moghadamtousi SZ, Kadir HA, Mohan G. Neuroinflammation pathways: a general review. Int J Neurosci. 2017;127:624-33.

41. Herrero MT, Estrada C, Maatouk L, Vyas S. Inflammation in Parkinson's disease: role of glucocorticoids. Front Neuroanat. 2015;9:32.

42. Whitmarsh AJ, Davis RJ. Transcription factor AP-1 regulation by mitogen-activated protein kinase signal transduction pathways. J Mol Med. 1996;74:589-607.

43. Wu J, Du J, Gu R, Zhang L, Zhen X, Li Y, et al. Inhibition of neuroinflammation by synthetic androstene derivatives incorporating amino acid methyl esters on activated BV-2 microglia. ChemMedChem. 2015;10:610-6.

44. Singh SS, Rai SN, Birla H, Zahra W, Rathore AS, Singh SP. NF-kappaB-mediated neuroinflammation in Parkinson's disease and potential therapeutic effect of polyphenols. Neurotox Res. 2020;37:491-507.

45. Goldberg MS, Pisani A, Haburcak M, Vortherms TA, Kitada T, Costa C, et al. Nigrostriatal dopaminergic deficits and hypokinesia caused by inactivation of the familial Parkinsonism-linked gene DJ-1. Neuron. 2005;45:489-96.

46. Guo DK, Zhu Y, Sun HY, Xu XY, Zhang S, Hao ZB, et al. Pharmacological activation of REV-ERBalpha represses LPS-induced microglial activation through the NFkappaB pathway. Acta Pharm Sin. 2019;40:26-34.

47. Tian LP, Zhang S, Xu L, Li W, Wang Y, Chen SD, et al. Selenite benefits embryonic stem cells therapy in Parkinson's disease. Curr Mol Med. 2012;12:1005-14.

48. Ren H, Fu K, Mu C, Zhen X, Wang G. L166P mutant DJ-1 promotes cell death by dissociating Bax from mitochondrial Bcl-XL. Mol Neurodegener. 2012;7:40.

49. Fu C, Chen D, Chen R, Hu Q, Wang G. The schizophrenia-related protein dysbindin-1A is degraded and facilitates NF-kappa B activity in the nucleus. PLoS ONE. 2015;10:e0132639.

50. Chen D, Li YP, Yu YX, Zhou T, Liu C, Fei EK, et al. Dendritic cell nuclear protein-1 regulates melatonin biosynthesis by binding to BMAL1 and inhibiting the transcription of N-acetyltransferase in C6 cells. Acta Pharm Sin. 2018;39:597-606.

51. Yu YX, Li YP, Gao F, Hu QS, Zhang Y, Chen D, et al. Vitamin K2 suppresses rotenone-induced microglial activation in vitro. Acta Pharm Sin. 2016;37:1178-89.

\section{ACKNOWLEDGEMENTS}

We would like to thank Dr. Jie Shen at Harvard Medical School to provide us the $D J-1$ knockout mice.

\section{AUTHOR CONTRIBUTIONS}

G.W. and H.R. performed study concept and design. H.R. wrote the paper. G.W. revised the paper. Z.L. performed the experiments, Z.L., C.C., D.Y., and J.D. analyzed the data. All authors read and approved the final paper.

\section{FUNDING}

This research was funded by the National Natural Science Foundation of China (31970966, 32070970, and 31871023), the National Key Scientific Research and Development Program of China (2016YFC1306000), Suzhou Clinical Research Center of Neurological Disease (Szzx201503), and the Priority Academic Program Development of Jiangsu Higher Education Institutions. 


\section{ETHICS APPROVAL}

The study does not involve human participants, human data, or human tissue. All mouse experiments were approved and carried out in accordance with the Regulations of Experimental Animal Administration, which are issued by the Animal Ethics Committee of Soochow University.

\section{COMPETING INTERESTS}

The authors declare no competing interests.

\section{ADDITIONAL INFORMATION}

Correspondence and requests for materials should be addressed to G.W. or H.R.

Reprints and permission information is available at http://www.nature.com/ reprints
Publisher's note Springer Nature remains neutral with regard to jurisdictional claims in published maps and institutional affiliations.

Open Access This article is licensed under a Creative Commons Attribution 4.0 International License, which permits use, sharing, adaptation, distribution and reproduction in any medium or format, as long as you give appropriate credit to the original author(s) and the source, provide a link to the Creative Commons license, and indicate if changes were made. The images or other third party material in this article are included in the article's Creative Commons license, unless indicated otherwise in a credit line to the material. If material is not included in the article's Creative Commons license and your intended use is not permitted by statutory regulation or exceeds the permitted use, you will need to obtain permission directly from the copyright holder. To view a copy of this license, visit http://creativecommons. org/licenses/by/4.0/.

(c) The Author(s) 202 